\title{
The Thermal Conductive Property of TDI based Thermoplastic Polyurethane/graphene Nanoplatelets Composite
}

\author{
Danqing Chen ${ }^{1, a^{*}}$, Jie Yan ${ }^{2, b}$ \\ ${ }^{1}$ Department of Polymer Science\&Engineering, Huaqiao University, Xiamen, 361021,China \\ ${ }^{2}$ Department of Mechanical Design\&Manufacturing, Huaqiao University, Xiamen, 361021, China \\ a chendan@hqu.edu.cn, byanjieer@hqu.edu.cn .
}

\begin{abstract}
Keywords: TDI based Thermoplastic Polyurethane, Graphene Nanoplatelets, Thermal Conductive Abstract. TDI based Thermoplastic polyurethane/graphene nanoplatelets(TPU/GNs) thermal conductive composite was in situ synthesised at room temperature, using polytetramethylene glycol(PEMG) as soft segment,4-Methyl-m-phenylene diisocyanate (TDI) as hard segment, 1,4-butanediol (BDO) as chain-extending agents, GNs as thermal conductive filler. AIN was used as contrast thermal conductive filler. The morphology, thermal conductive properties of TPU/GNs composite were investigated. It showed that the percolation threshold effect of TPU/GNs composite occurred at the content around $12 \mathrm{wt} \%$ of the GNs, and the thermal conductive properties was better than TPU/AIN composite. Besides, TDI based TPU thermal conductive composite showed much better thermal conductive properties while the thermal conductive filler was $10 \%$ GNs plus $2 \%$ AIN for the synergistic effect.
\end{abstract}

\section{Introduction}

TDI based Thermoplastic polyurethane (TPU) is a kind of environmental friendly materials with characteristics of nontoxic, recyclable and excellent physical chemical properties, widely used in mining, oil field, toys, pipes, shoes, clothes, automobile and so on. TDI based TPU's main raw materials include diisocyanates, macrodiols and diol. The main macrodiols include polytetramethylene glycol(PEMG), polypropylene oxide(PPO) and polycaprolactone (PCL) and so on. The main diol include 1,4-butanediol (BDO) , 1,6-hexanediol (HG) and ethylene glycol (EG) and so on. TDI, PEMG and BDO are widely used to synthesize TPU due to the moderate cost and simple maneuverability (can be operated at room temperature for the low melt point). However, Its thermal conductivity is very low(about $0.2 \mathrm{w} / \mathrm{m} . \mathrm{k}$ ), cannot be effective release the high quantity of heat, so the application in the field of electronic information is limited. M. Vezir Kahraman found that thermal conductivity of the TPU/h-BN composite materials was improved 33\% with the incorporation of $5 \mathrm{wt} \%$ nano h-BN, but the thermal conductive was still poor [1]. GNs, is also called as Graphite nanosheets, has advantages in forming electric conducting networks in polymer matrices such as PMMA[2], PS [3] ,epoxy [4], unsaturated polyester [5],HDPE[6], silicone rubber [7] and PU foams [8] for the high aspect ratio. GNs will also has advantages in forming thermal conducting networks in polymer matrices. In this paper, TPU/GNs thermal conductive composite was in situ synthesised at room temperature using GNs as conductive filler, TDI as hard segment, PEMG as soft segment, BDO as chain-extending agents.

\section{Experimental}

\section{Materials}

GNs with thickness of $30 \sim 80 \mathrm{~nm}$, diameter of $5 \sim 20 \mu \mathrm{m}$, were provided by Xiamen KNANO Graphene Technology Corporation Limited (China). AIN 、4-Methyl-m-phenylene diisocyanate (TDI) and Catalyst were provided by Fujian BAICHANG Polyurethane Corporation Limited (China). Polytetramethylene glycol 
(PEMG, Mw:1000) and 1,4-butanediol(BDO) were provided by Aladdin Chemistey Corporation Limited (China).

\section{Preparation of TPU/GNs thermal conductive composite}

The TDI based TPU/GNs thermal conductive composite was in situ synthesised at room temperature. The formula was devised as $\mathrm{n}(\mathrm{PEMG}) \cdot \mathrm{na}(\mathrm{BDO}): \mathrm{n}(\mathrm{a}+1)(\mathrm{TDI})=1: 2: 3, \quad \mathrm{R}=\mathrm{n}(\mathrm{NCO}) \cdot \mathrm{n}(\mathrm{OH})=1.05$ (TPU will crosslink seriously if $\mathrm{R}>1.05$, will react incompletely if $\mathrm{R}<1.05$ ). The process of in situ synthesis of TPU/GNs conductive composite was shown in Figure 1. Firstly, the premixture with GNs, 10g PEMG(0.01mol), $1.8 \mathrm{gBDO}(0.02 \mathrm{~mol})$, mixed with $5.5 \mathrm{~g} \mathrm{TDI}(0.0315 \mathrm{~mol})$ and $0.05 \mathrm{~g}$ catalyst in the condition of $1500 \mathrm{rpm}$ for about $3 \mathrm{~min}$ using stirrer, poured into a mold to react at $120^{\circ} \mathrm{C}$ in vacuumoven(avoid foaming) for $12 \mathrm{hr}$. Lastly, the resulted composite slices were directly removed from the mold before the subsequent testing. The TPU/AIN composite was prepared in the similar process as contrast.

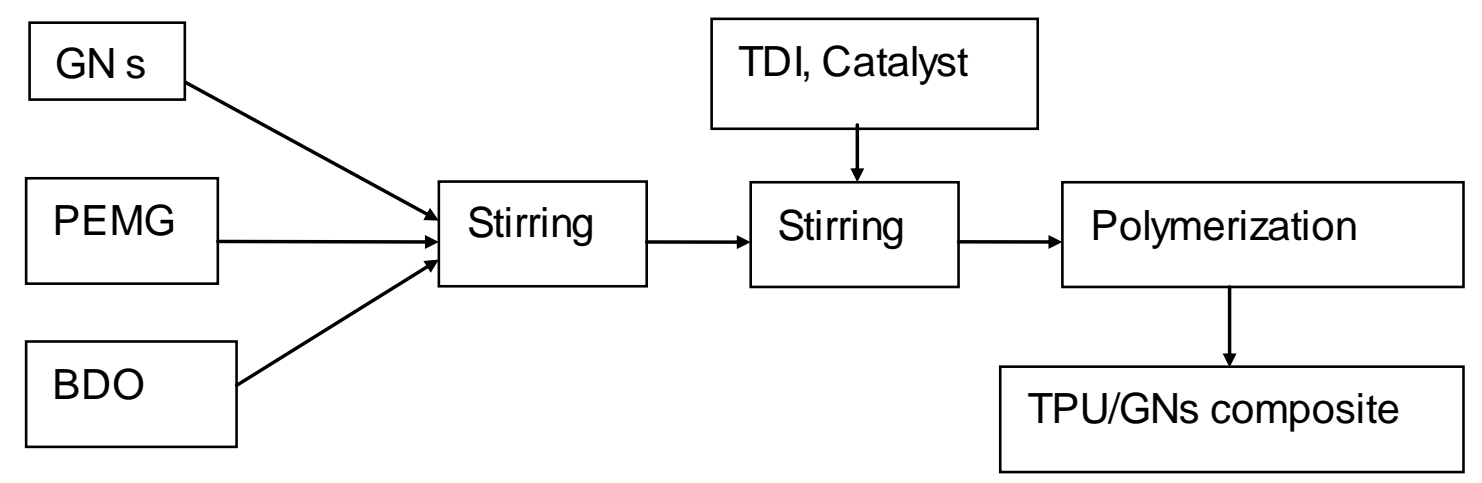

Fig.1 Process of in situ synthesis of TDI based TPU/GNs thermal conductive composite

\section{Testing of Sample}

Scanning electron microscopy (SEM) was performed using a HITACHI S-4800 field-emitting scanning electron microscope at an operating voltage of $5 \mathrm{KV}$, the surfaces were vacuum coated with a thin gold layer before testing. The thermal conductivity $(\mathrm{k}, \mathrm{W} / \mathrm{m} . \mathrm{K})$ of the samples (diameter: $12.7 \mathrm{~mm}$, thickness: about 2 $\mathrm{mm}$ ) was calculated by the product of thermal diffusivity $\left(\delta, \mathrm{mm}^{2} / \mathrm{s}\right)$, specific heat $(\mathrm{C}, \mathrm{J} / \mathrm{g} \cdot \mathrm{K})$, and bulk density $(\rho, \mathrm{g} / \mathrm{cm} 3): \mathrm{k}=\delta \times \mathrm{C} \times \rho(1)$ where $\delta$ and $\mathrm{C}$ were measured using an LFA447 light flash system (NETZSCH, Germany). Before testing, we also sprayed the Graphite coating on the sample's surface following the LFA447 light flash system's request.

\section{Results}

\section{Morphological analysis}

The morphology of the TDI based TPU / GNs composites with $3 \mathrm{wt} \% \mathrm{GNs}$ in situ synthesised is shown in Fig. 2. It can be seen that GNs can well located in the TPU phase. 


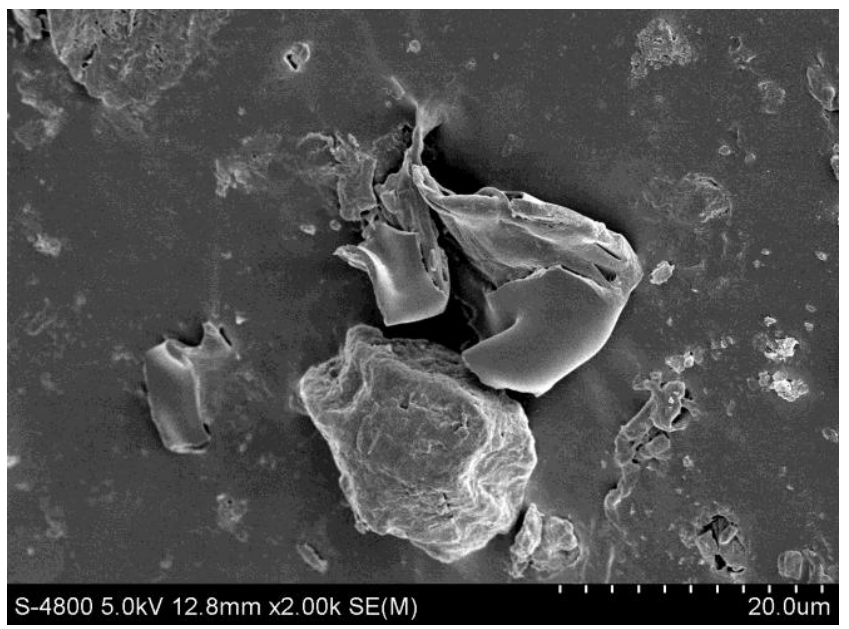

Fig.2 SEM micrographs of TPU/ GNs composites in situ synthesised

\section{Thermal conductive properties}

The thermal conductivity of the TDI based TPU /GNs composite as a function of the GNs weight content is shown in Fig.3. The addition of conducting fillers and temperature significantly increase the thermal conductivity of composite. The percolation threshold effect of TPU/GNs composite occurred at the content around $12 \mathrm{wt} \%$ of the GNs While the raw material of the composite cann't be mixed uniform if the filler's content is more than $15 \%$, so we have not gone beyond the particular value. The above results is attributed to the much high aspect ratio of GNs, sheet-like filler will result in forming thermal conducting networks in polymer matrices.

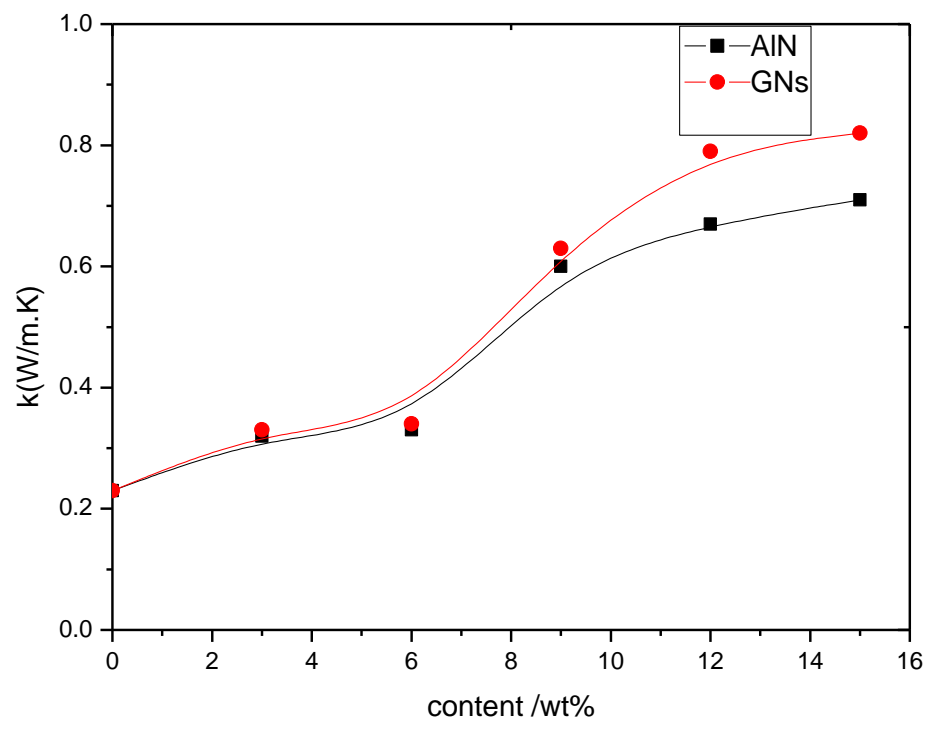

Fig.3 Thermal conductivity of TPU composite as a function of filler's content

Besides, Thermal conductivity of TPU composite as a function of GNs content(total filler is $12 \%$ ) is shown in Fig.4.TDI based TPU thermal conductive composite showed much better thermal conductive properties while the thermal conductive filler was $10 \%$ GNs+2\% AIN for the synergistic effect. 


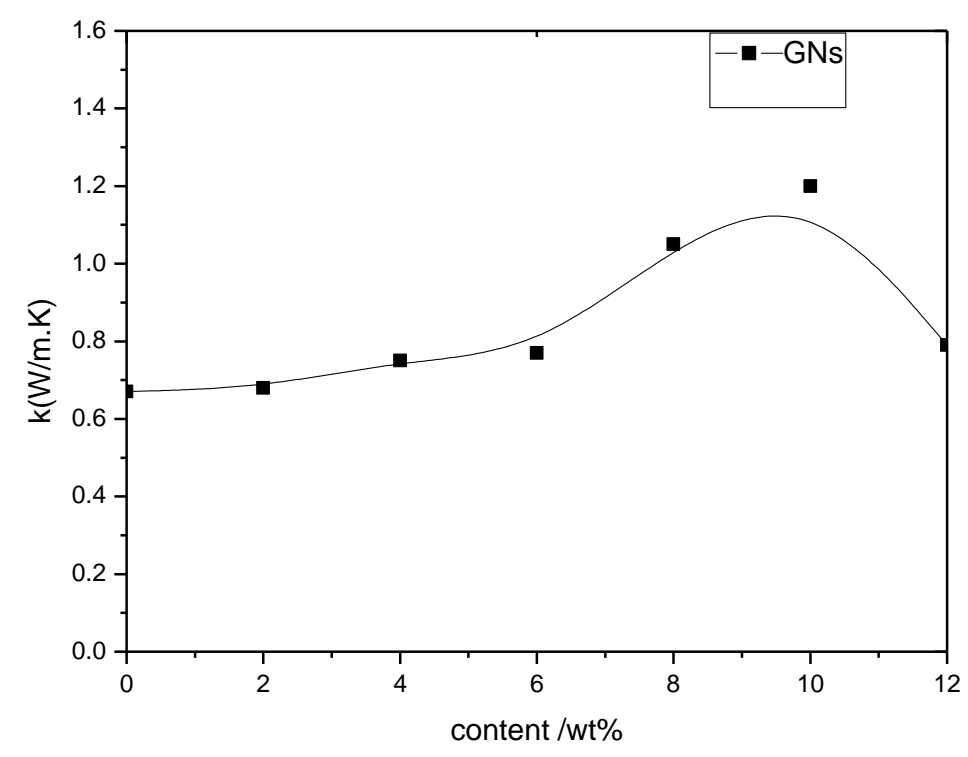

Fig.4 Thermal conductivity of TPU composite as a function of GNs content(total filler is $12 \%$ )

\section{Summary}

TDI based TPU/GNs thermal conductive composite was in situ synthesise at room temperature, using GNs as thermal conductivity filler. GNs can well located in the TPU phase, the percolation threshold was about $12 \mathrm{wt} \%$, the thermal conductive properties was better than TPU/AIN composite. Besides, TDI based TPU thermal conductive composite showed much better thermal conductive properties while the thermal conductive filler was $10 \%$ GNs plus $2 \%$ AIN. Due to the moderate cost and simple maneuverability and good property, this kind of TDI based TPU/GNs thermal conductive composite can be widely used in electronics in future.

\section{Acknowledgement}

This work is supported by the Science Foundation of Xiamen(3502Z20143044) and the Science Foundation of Huaqiao University(14BS117).

\section{References}

[1] Emrah $\mathrm{C}_{3}$ akmakc, M.Vezir Kahraman,et al,Preparation and Characterization of Thermally

Conductive Thermoplastic Polyurethane/h-BN Nanocomposites,POLYM. COMPOS., 35(2014) $530-538$.

[2] Chen Guohua,Wu Dajun,et al, Exfoliation of graphite flake and its nanocomposites, Carbon, 41 (2003)619-621.

[3] Chen Guohua,Wu Cuiling,et al, Preparation of polystyrene/graphite nanosheet composite, Polymer,44(2003)1781-1784. 
[4] Lu Wei,Weng Jianxin,Chen Guohua,et al, Epoxy Resin/Graphite Nanosheets Electrically Conductive Nanocomposite,Materials and Manufacturing Processes, 21(2006)167-171.

[5] Chen Guohua, Wang Haiquan, Zhao Weifeng, Fabrication of Highly Ordered Polymer/Graphite Flake Composite with Eminent Anisotropic Electrical Property,Polymers for Advanced Technologies, 19(2008)1113-1117.

[6] LiChun Zhou,Jinshan Lin, Guohua Chen, Electrical Breakdown in High-Density Polyethylene /Graphite Nanosheets Conductive Composites, Journal of Polymer Science: Part B: Polymer Physics, 47(2009)576-582.

[7] Ling Chen, Guohua Chen, Relaxation Behavior Study of Silicone Rubber Crosslinked Network Under Static and Dynamic Compression by Electric Response,POLYM. COMPOS., 30(2009) 101-106. [8] Danqing Chen, Guohua Chen, et al, The physical properties of polyurethane/graphite nanosheets /carbon black foaming conducting nanocomposites, Composites: Part A. 41(2010) 1636-1638 